\title{
Predictors of Survival after Total Laryngectomy for Recurrent/Persistent Laryngeal Squamous Cell Carcinoma
}

Andrew C. Birkeland, M.D., Lauren Beesley M.S., Emily Bellile M.S., Andrew J. Rosko, M.D, Rebecca Hoesli, M.D., Steven B. Chinn, M.D., Andrew G. Shuman, M.D., Mark E. Prince, M.D., Gregory T. Wolf, M.D., Carol R. Bradford, M.D., J. Chad Brenner, Ph.D., Matthew E. Spector, M.D.

Department of Otolaryngology -Head and Neck Surgery, University of Michigan Health System, Ann Arbor, Ml 48109

Funding: Dr. Birkeland was funded with an NIH T32 DC005356 and a University of Michigan Otolaryngology Resident Research Grant. Ms. Beesley was funded with an NIH T32 CA-83654 grant.
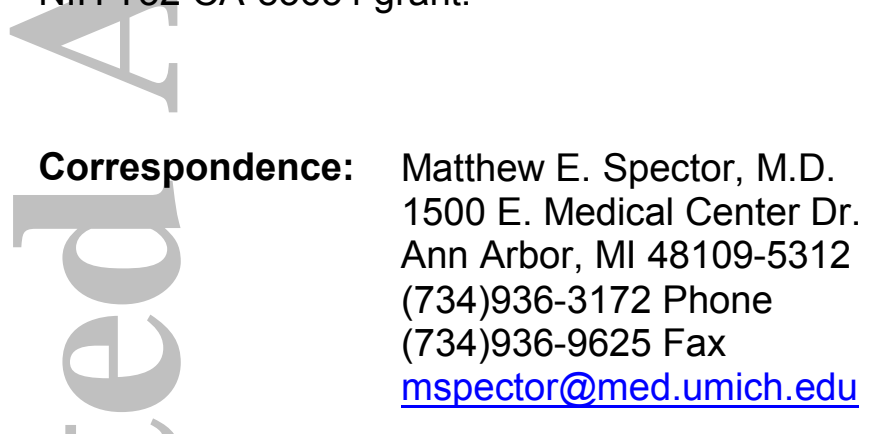

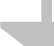

Running Title: $\quad$ Survival in Recurrent Larynx Cancer

Keywords:

Recurrent laryngeal cancer, salvage laryngectomy, overall survival, disease specific survival, ACE-27

This is the author manuscript accepted for publication and has undergone full peer review but has not been through the copyediting, typesetting, pagination and proofreading process, which may lead to differences between this version and the Version record. Please cite this article as doi:10.1002/ hed.24918. 


\section{Abstract}

Background:Total laryngectomy remains the treatment of choice for recurrent/persistent laryngeal squamous cell carcinoma(LSCC) after radiation(RT) or chemoradiation(CRT). However, despite attempts at aggressive surgical salvage, survival in this cohort remains suboptimal.

Methods:A prospectively-maintained single-institution database was queried for patients undergoing total laryngectomy for recurrent/persistent LSCC after initial RT/CRT between 1998-2015(n=244). Demographic, clinical, and survival data were abstracted. Kaplan Meier survival curves and hazard ratios(HR) were calculated.

Results:Five-year overall survival was $49 \%$. Five-year disease-free survival was $58 \%$. Independent predictors of overall survival included severe comorbidity[ACE-27

scale](HR 3.76; 95\% Cl 1.56 - 9.06), and positive recurrent clinical nodes(HR 2.91; 95\% Cl $1.74-4.88)$.

Conclusion:Severe comorbidity status is the strongest predictor of overall survival, suggesting that increased attention to mitigating competing risks to health is critical. These data may inform a risk prediction model to allow for focused shared decisionmaking, preoperative health optimization and patient selection for adjuvant therapies.

John Wiley \& Sons, Inc.

This article is protected by copyright. All rights reserved. 


\section{Introduction:}

Advanced laryngeal squamous cell carcinoma (LSCC) remains a highly morbid and fatal disease despite aggressive interventions. Mortality is high in this cohort: larynx cancer affects over 13,000 patients in the United States, with over 3,500 deaths annually ${ }^{[1]}$. The results of the VA Cooperative Studies Program Laryngeal Cancer Trial and subsequent studies demonstrated similar overall survival rates for organ preservation protocols involving radiation (RT) or chemoradiation (CRT) when compared to surgery, with the benefit of preserving the larynx in a large majority of patients ${ }^{[2-5]}$. As such, the use of RT or CRT has become the predominant initial intervention for patients with LSCC $^{[6]}$. However, the prognosis of recurrent and persistent LSCC is particularly dire.

Notably, a significant subset of patients undergoing RT or CRT for LSCC will develop recurrent disease. Five-year disease-free survival rates for advanced LSCC treated with organ preservation protocols ranges from $30-60 \%{ }^{[3,5,7]}$; for most with

recurrent/persistent disease, salvage laryngectomy is often the only remaining curative therapeutic modality. Despite this, 5 -year overall survival remains poor and is further compounded by a short disease-free interval ${ }^{[8]}$. The morbidity of salvage surgery in such patients is considerable; moreover, survival for patients with recurrent or persistent LSCC is poor, but incompletely characterized ${ }^{[9-11]}$.

To date, there have not been studies focused on assessing prognostic variables for patients considering salvage laryngectomy. While overall survival and recurrence rates are well described for primary LSCC, there is a paucity of data assessing outcomes for patients undergoing salvage laryngectomy. As organ-preservation protocols are increasingly being implemented as first-line therapy and failure drives poor survival, this patient population will be increasingly important to consider. Likewise, given recent

John Wiley \& Sons, Inc.

This article is protected by copyright. All rights reserved. 
increases in personalized medicine and immunotherapy approaches for advanced head and neck squamous cell carcinomas ${ }^{[12,13]}$, it is critical to identify predictors for LSCC patients that may benefit from the addition of neo/adjuvant therapy in conjunction with salvage surgery.

We sought to identify preoperative predictors of survival for patients with recurrent or persistent LSCC in order to set patient expectations and address modifiable risk factors. Identification of predictors of survival may also identify patients who could benefit from novel therapeutic agents in a neo/adjuvant fashion. Herein, we examine a cohort of patients with recurrent or persistent LSCC after definitive RT/CRT that have undergone total laryngectomy in order to identify potential preoperative predictors of clinical outcomes.

\section{Materials and Methods:}

Patient Identification and Data Collection

A prospectively-maintained single-institution epidemiology database of patients with head and neck cancer was queried for eligible patients. University of Michigan Institutional Review Board approval was obtained for this study (HUM00081554). Inclusion criteria specified adult patients with a diagnosis of LSCC initially treated with RT/CRT between 1998-2015, who had a salvage laryngectomy for recurrent/persistent disease at the primary site $(n=244)$. Demographic, clinical, and survival data were abstracted (Table 1). Death was verified via medical records and the social security death index. Primary outcome measures were overall survival (OS; time from salvage laryngectomy to death from any cause), disease-specific survival (DSS; time from salvage laryngectomy to death from recurrent/persistent LSCC), and disease-free survival (DFS; time from salvage laryngectomy to LSCC recurrence). Survival was described with Kaplan-Meier methodology. We focused our analysis on survival 
patterns during the first five years after salvage surgery. For comorbidities, the ACE-27 index was used as previously described ${ }^{[14]}$, with comorbidity status classified as none, mild, moderate, and severe.

Preoperative data (Table 1) included patient demographics, smoking history (defined as current, former: quit over 1 year ago, or never), comorbidity status (ACE-27 scale), initial cancer characteristics, initial cancer treatment, and recurrent cancer clinical characteristics.

\section{Statistical Analysis}

We aimed to identify factors at the time of the preoperative evaluation, as this would be a key timepoint in which to discuss patient care options and prognosis. Thus, we included variables available to clinicians at the preoperative appointment for recurrent/persistent LSCC. We first calculated summary statistics to describe the analytical sample of $\mathrm{N}=244$ patients with recurrent laryngeal cancer. The Kaplan-Meier method was used to estimate survival curves for OS, DFS, and DSS. We then estimated the predicted state probabilities (alive, died from cancer, died from other causes) over time broken down by comorbidity level. The probability of death from any cause was estimated using the Kaplan-Meier method, and the probability of death specifically from other causes was estimated using Cumulative Incidence, which accounts for the competing risks between death from other causes and death from cancer. To identify additional preoperative factors associated with OS, DSS, and DFS, we fit a multivariable Cox proportional hazards regression model for each outcome. Due to the large number of predictors, we used forward selection to identify the most important covariates for each model. All analyses were performed using R version 3.3.0 (Vienna, Austria).

\section{Results:}


30-day Mortality after Laryngectomy for Recurrent/Persistent LSCC

The 30-day mortality for the entire cohort was $5 \%(13 / 244)$. Cause of death in this cohort included cardiopulmonary arrest (3), carotid blowout (2), metastatic disease (2), stroke (1), bowel ischemia (1), pulmonary embolus (1), respiratory failure (1), infection (1), and unknown, not larynx cancer (1). To understand the association between common perioperative morbidity and 30-day mortality, we compared fistula rate, return to the operating room for management of fistula/wound issues, and ACE-27 comorbidity status. Our fistula rate was $34 \%$ (84/244; Table 2). Our operative takeback rate was $12 \%(30 / 244)$. There was no association with the development of a fistula or return to the operating room and 30-day mortality. There was an increasing and significantly worsened 30-day mortality for patients with mild/moderate and severe comorbidity status.

Survival Analysis after Laryngectomy for Recurrent/Persistent LSCC In Kaplan Meier analysis, the estimated five-year OS was $49 \%$ within the entire cohort (95\% confidence interval [CI] 42-56 \%; Figure 1A). Five-year DSS for the entire cohort was $68 \%$ (95\% Cl 61-75\%; Figure 1B). Five-year DFS for the cohort was $59 \%(95 \% \mathrm{Cl}$ 52-67\%; Figure 1C). We next stratified the cohort by type of recurrence (locoregional versus distant). In Kaplan Meier analysis, the estimated five-year locoregional DFS was $71 \%$ (95\% Cl: $65 \%$ - 78\%). Five-year distant DFS was $78 \%$ (95\% Cl $71 \%-85 \%)$.

\section{Multivariable Modeling of Preoperative Predictors of Survival}

We performed multivariable analysis in order to predict variables contributing to OS, DSS, and DFS. Using a forward step model, we included demographic (gender, ethnicity, ACE-27 comorbidity status, tobacco use), initial tumor characteristic (age at initial tumor, initial site, initial cT classification, initial cN classification, initial overall

John Wiley \& Sons, Inc.

This article is protected by copyright. All rights reserved. 
stage, initial treatment, initial treatment location), and recurrent tumor characteristic (age at recurrence, time to recurrence, recurrent site, recurrent cT classification, recurrent $\mathrm{cN}$ classification, recurrent overall stage; Table 1) variables. In multivariable analysis, significant preoperative predictors of OS (Table 3) included severe comorbidity [ACE-27 scale] (HR 3.76; 95\% Cl 1.56 - 9.06), recurrent clinical node positive status (HR 2.91; $95 \% \mathrm{Cl} 1.74-4.88)$, and initial overall stage greater than I. For DSS, predictors included recurrent clinical node positive status (HR 3.95; 95\% Cl 2.16-7.22), and initial overall stage greater than I. Multivariable independent predictors of DFS (Table 3) included recurrent clinical node positive status (HR 3.26; $95 \% \mathrm{Cl} 1.83-5.78$ ), and initial overall stage greater than I.

To better assess the preoperative effect size of medical comorbidities on overall survival, we further stratified causes of death between cancer and non-cancer (namely, comorbidity) causes over time (Figure 2A-C). Non-cancer causes of death contributed to significant decrease overall survival over a 5 -year period in our overall cohort. This was particularly evident in patients with moderate or severe comorbidities (Figure 2B), more than those with mild comorbidities (Figure 2A). In analysis of rate of cancer versus non-cancer causes of death, for patients with moderate or severe comorbidities, non-cancer causes of death (i.e. comorbid conditions) was a greater cause of death than cancer-related causes through 30 months after salvage laryngectomy (Figure 2C). From 30 months to 5 years, patients with moderate or severe comorbidity status had an equal probability of dying from their comorbidities as their LSCC. Cause of death for patients with no or mild comorbidity status is more likely to be due to a recurrence of disease.

\section{Discussion:}


The prognosis after laryngectomy for recurrent/persistent LSCC remains guarded; we observed a 5 -year OS probability of $49 \%$. Severe comorbidity status is the strongest independent predictor thereof, suggesting that careful preoperative discussions and increased attention to mitigating competing risks to health is critical in this population. Overall, in our cohort, we found that the strongest independent predictors included severe comorbidity and recurrent clinical node positive status for overall survival, and clinical node positive status for disease-free survival. These data may allow for focused shared decision-making between patient and physician, and patient selection for potential adjuvant therapies.

To date, there have been no multivariable analyses for prognostic factors for patients undergoing salvage laryngectomy. For patients and physicians alike, identification of patients who may have poor predicted survival after salvage laryngectomy will be important to guide physician-patient discussions on goals of care, expectations and follow-up, and to consider for potential adjuvant or investigational treatment.

Increasingly, management of comorbidities and chronic care are becoming key issues in the survivorship phase of head and neck cancer management ${ }^{[15]}$. Our data support that increased comorbidities have a significant effect on overall survival in patients undergoing salvage laryngectomy. Thus, strong consideration of patients' competing risks of mortality in the preoperative setting may frame a realistic discussion on overall survival. Additionally, there may be a role for active interventions to modify the lifelimiting impact of specific comorbidities (e.g. medications for depression or hypertension, treatment of cardiovascular and pulmonary disease, counseling on alcohol or preoperative smoking cessation). Common modifiable risk factors included in the ACE- 
27 score include poorly controlled diabetes, hypertension, depression, obesity, and alcohol abuse.

Interestingly, smoking status did not demonstrate significance on multivariable analysis.

We postulate that the extensive smoking history of our patients with cumulative effects of smoking and almost universal smoking cessation after laryngectomy may explain any lack of effect on current smoking status on survival. Additionally, the effects of smoking may be best reflected on the severity of comorbidities collected by ACE-27

(cardiovascular, respiratory, neurologic disease, and malignancy), thus making ACE-27

comorbidity status a more comprehensive factor in accounting for the effects of smoking on a patient's survival. Similarly, we did not see significance of clinical T classification on multivariable analysis. Although advanced clinical T classification was associated with a worse outcome on univariable analysis, it was also correlated with clinical nodal positivity. We postulate that this correlation and other variables (initial tumor factors, time to recurrence factors), may account for the lack of effect in multivariable analysis.

Our analysis did not show any survival difference between initial treatment between initial RT or CRT at our institution versus outside hospitals. In regards to outside hospital treatment, the University of Michigan collaborates closely with local hospitals in regards to radiation planning, in many cases assisting in the radiation fields to be implemented. In addition, patient's treated at the University of Michigan were more like to have supraglottic cancers (62\% UM vs $36 \%$ OSH) and advanced stage cancers ( $57 \%$ UM vs $29 \% \mathrm{OSH}$ ), which may explain the lack of significance.

Counseling and surgical decision-making when facing salvage surgery should emphasize the significant effect of comorbidities on survival, as well as the suboptimal

John Wiley \& Sons, Inc.

This article is protected by copyright. All rights reserved. 
observed survival within this cohort. Furthermore, counseling in regards to optimizing modifiable comorbidities (e.g. improved control of hypertension or COPD), may have valuable effects in a patient's estimated 5-year survival. Honest discussions about patient expectations and outcomes may guide further treatment plans. Our data demonstrates increasingly worsening 30-day perioperative mortality with increasing comorbidity status, suggesting a need for careful preoperative patient stratification, risk reduction of modifiable comorbidity factors, and counseling on perioperative complications and survival.

Despite our study's strength as the largest cohort of patients with recurrent/persistent LSCC undergoing salvage laryngectomy, it does have limitations. Initial tumor staging and treatment data was limited in some instances due to limited outside hospital records. The majority of our patients have their outside records sent to our institution, and staging reviewed at our tumor board, but in some cases this could be suboptimal. However, this is a common clinical scenario for patients presenting with recurrent or persistent LSCC to tertiary cancer centers. The vast majority of our patients had neck dissections, as this is standard practice amongst most of the surgeons in our institution ( $n=225 / 244)$; no survival difference was seen with the performance of neck dissection. The importance of neck dissection with salvage laryngectomy remains debated ${ }^{[16]}$, and some institutions do not perform neck dissections regularly. Further multi-institutional investigation by accruing survival data from patients with and without neck dissection may provide definitive data on this topic.

Patients undergoing salvage laryngectomy have a guarded prognosis. By stratifying based upon preoperative risk factors, we are able to define which subset of patients may have better or worse predicted outcomes. This information may be crucial to guide 
preoperative discussions on goals of care and consideration of adjuvant treatment modalities. As cancer care increasingly implements genetic biomarkers and targeted therapies, we must continually consider additional methodologies for risk stratification. Further validation of these predictive variables across additional cohorts will be invaluable to further refine management algorithms.

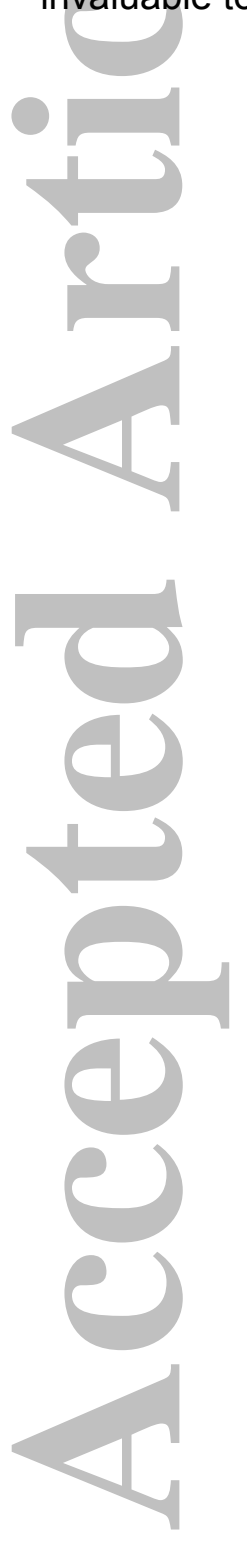

John Wiley \& Sons, Inc.

This article is protected by copyright. All rights reserved. 


\section{References:}

[1] Siegel, RL, Miller, KD., Jemal, A.Cancer statistics, 2015. CA Cancer J Clin, 2015. 65(1): p. 5-29.

[2] Induction chemotherapy plus radiation compared with surgery plus radiation in patients with advanced laryngeal cancer. The Department of Veterans Affairs Laryngeal Cancer Study Group. N Engl J Med, 1991. 324(24): p. 1685-90.

[3] Forastiere, AA, Goepfert, H., Maor, M., et al., Concurrent chemotherapy and radiotherapy for organ preservation in advanced laryngeal cancer. N Engl J Med, 2003. 349(22): p. 2091-8.

[4] Lefebvre, JL, Andry, G., Chevalier, D., et al., Laryngeal preservation with induction chemotherapy for hypopharyngeal squamous cell carcinoma: 10-year results of EORTC trial 24891. Ann Oncol, 2012. 23(10): p. 2708-14.

[5] Forastiere, AA, Zhang, Q., Weber, RS., et al., Long-term results of RTOG 91-11: a comparison of three nonsurgical treatment strategies to preserve the larynx in patients with locally advanced larynx cancer. J Clin Oncol, 2013. 31(7): p. 84552.

[6] Megwalu, UC, Sikora, AG. Survival outcomes in advanced laryngeal cancer. JAMA Otolaryngol Head Neck Surg, 2014. 140(9): p. 855-60.

[7] Denaro, N., Russi, EG., Lefebvre JL., Merlano MC. A systematic review of current and emerging approaches in the field of larynx preservation. Radiother Oncol, 2014. 110(1): p. 16-24.

[8] Sandulache, VC, Vandelaar, LJ., Skinner, HD., et al., Salvage total laryngectomy after external-beam radiotherapy: A 20-year experience. Head Neck, 2016. 38 Suppl 1: p. E1962-8.

[9] Davidson, J., Keane, T., Brown, D., et al., Surgical salvage after radiotherapy for advanced laryngopharyngeal carcinoma. Arch Otolaryngol Head Neck Surg, 1997. 123(4): p. 420-4.

[10] Brenner, B., Marshak, G., Sulkes, A., Rakowsky, E., , Prognosis of patients with recurrent laryngeal carcinoma. Head Neck, 2001. 23(7): p. 531-5.

[11] Li, P., Hu, W., Zhu, Y., Liu, J. Treatment and predictive factors in patients with recurrent laryngeal carcinoma: A retrospective study. Oncol Lett, 2015. 10(5): p. 3145-3152.

[12] Ludwig, ML, Birkeland, AC., Hoesli, R., Swiecicki, P., Spector, ME., Brenner, JC. Changing the paradigm: the potential for targeted therapy in laryngeal squamous cell carcinoma. Cancer Biol Med, 2016. 13(1): p. 87-100.

[13] Birkeland, AC, Yanik, M., Tillman, BN., et al., Identification of Targetable ERBB2 Aberrations in Head and Neck Squamous Cell Carcinoma. JAMA Otolaryngol Head Neck Surg, 2016. 142(6): p. 559-67.

[14] Piccirillo, JF, Tierney, RM., Costas, I., Grove, L., Spitznagel, EL Jr. Prognostic importance of comorbidity in a hospital-based cancer registry. JAMA, 2004. 291(20): p. 2441-7.

[15] Cohen, EE, LaMonte, SJ., Erb, NL., et al., American Cancer Society Head and Neck Cancer Survivorship Care Guideline. CA Cancer J Clin, 2016. 66(3): p. 203-39.

[16] Birkeland, AC, Rosko, AJ., Issa, MR. et al., Occult Nodal Disease Prevalence and Distribution in Recurrent Laryngeal Cancer Requiring Salvage Laryngectomy. Otolaryngol Head Neck Surg, 2016. 154(3): p. 473-9. 
Figure 1. 5-year Kaplan Meier Survival Curves . 5-year survival patterns demonstrate an overall survival of $49 \%(\mathbf{A} ; 95 \% \mathrm{Cl} 42-56 \%)$, a disease-specific survival of $68 \%(B ; 95 \% \mathrm{Cl} 61-75 \%$ ), and disease-free survival of $59 \%$ (C; $95 \% \mathrm{Cl} 52-67 \%$ ).
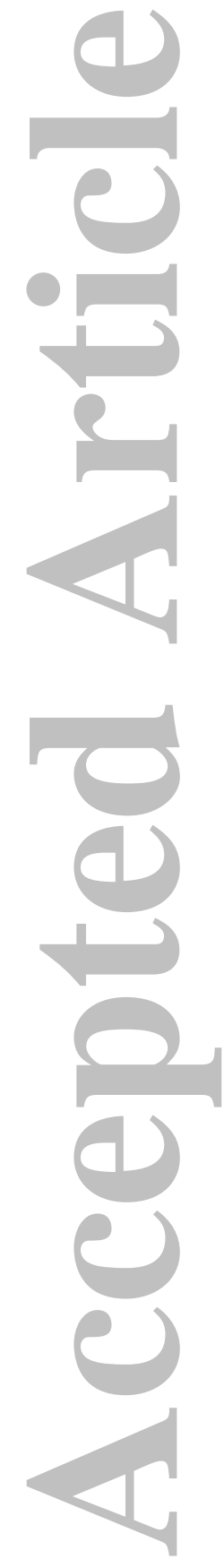

John Wiley \& Sons, Inc.

This article is protected by copyright. All rights reserved. 
Figure 2. Contribution of Comorbidity Status to Overall Survival. Cumulative proportion of subjects who died from cancer and non-cancer causes over 5 years (A).

Patients with moderate or severe comorbidities are more likely to die from their comorbidities and other causes than cancer through 30 months $(\mathbf{B}, \mathbf{C})$. The $y$-axis is the ratio of probability of dying from LSCC versus non-cancer causes. Ratio of probability at 1 (horizontal line) denotes an equal probability of dying from non-cancer causes as dying from cancer. Lines below 1 suggest a greater risk of dying from non-cancer causes and lines above 1 denote greater risk of dying from LSCC (C).

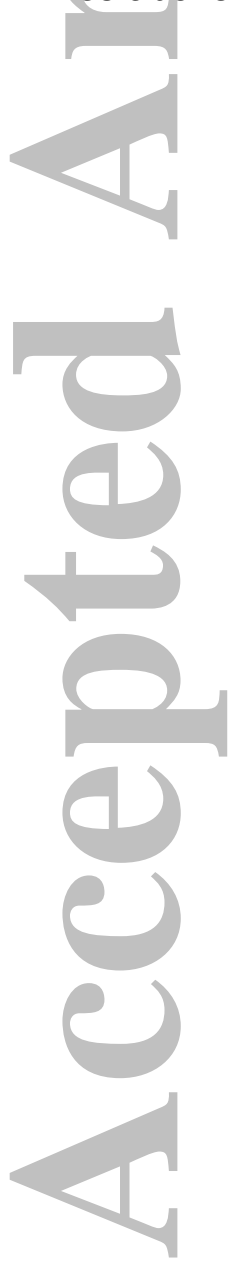

John Wiley \& Sons, Inc.

This article is protected by copyright. All rights reserved. 

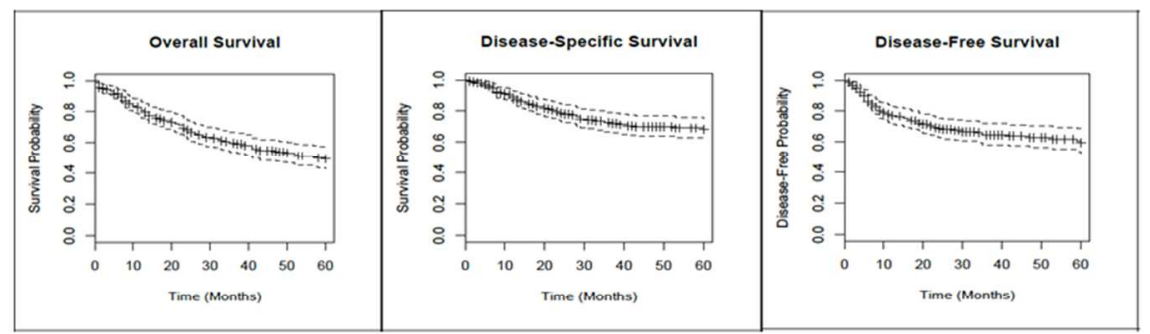

Figure 1. 5-year Kaplan Meier Survival Curves . 5-year survival patterns demonstrate an overall survival of $49 \%(A ; 95 \%$ CI $42-56 \%$ ), a disease-specific survival of $68 \%(B ; 95 \%$ CI $61-75 \%)$, and disease-free survival of $59 \%$ (C; $95 \%$ CI $52-67 \%$ ).

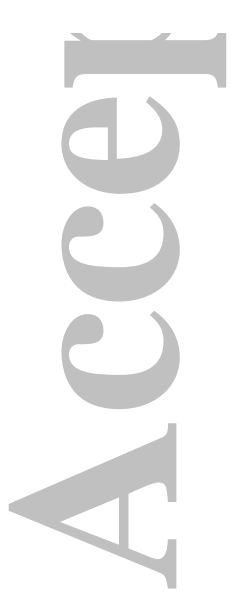

$254 \times 190 \mathrm{~mm}(96 \times 96 \mathrm{DPI})$

John Wiley \& Sons, Inc.

This article is protected by copyright. All rights reserved. 

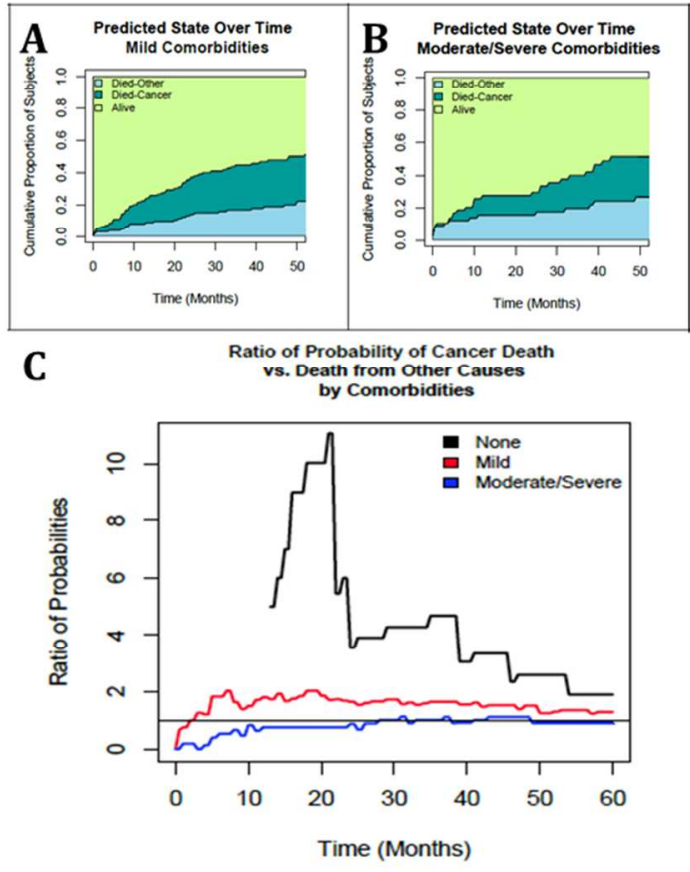

Figure 2. Contribution of Comorbidity Status to Overall Survival. Cumulative proportion of subjects who died from cancer and non-cancer causes over 5 years (A). Patients with moderate or severe comorbidities are more likely to die from their comorbidities and other causes than cancer through 30 months $(B, C)$. The $y$ axis is the ratio of probability of dying from LSCC versus non-cancer causes. Ratio of probability at 1 (horizontal line) denotes an equal probability of dying from non-cancer causes as dying from cancer. Lines below 1 suggest a greater risk of dying from non-cancer causes and lines above 1 denote greater risk of dying from LSCC (C).

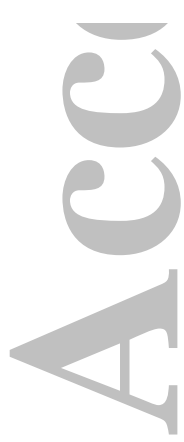

$254 \times 190 \mathrm{~mm}(96 \times 96 \mathrm{DPI})$

John Wiley \& Sons, Inc.

This article is protected by copyright. All rights reserved. 
Table 1. Cohort Characteristics $(n=244)$

\begin{tabular}{|c|c|c|}
\hline & $\begin{array}{l}\mathbf{N}(\%) \\
\text { Or Mean (SD) }\end{array}$ & Missing, $\mathbf{N}(\%)$ \\
\hline $\begin{array}{l}\text { Patient Characteristics } \\
\text { Gender } \\
\text { Male } \\
\text { Female } \\
\text { Ethnicity } \\
\text { White } \\
\text { Black/Other/Unknown } \\
\text { Comorbidities } \\
\text { None } \\
\text { Mild } \\
\text { Moderate } \\
\text { Severe } \\
\text { Tobacco Use } \\
\text { Current } \\
\text { Former } \\
\text { Never }\end{array}$ & $\begin{array}{l}208(85.2) \\
36(14.7) \\
223(91.3) \\
21(8.6) \\
\\
49(20.0) \\
129(52.8) \\
51(20.9) \\
15(6.1) \\
92(37.7) \\
146(59.8) \\
6(2.4)\end{array}$ & $0(0)$ \\
\hline $\begin{array}{l}\text { Initial Cancer } \\
\text { Age at Initial Tumor } \\
\text { Initial Site } \\
\text { Glottis } \\
\text { Supraglottis } \\
\text { Subglottis } \\
\text { Initial cT Classification } \\
\text { cT1 } \\
\text { cT2 } \\
\text { cT3 } \\
\text { cT4 } \\
\text { Initial cN Classification } \\
\text { cN0 } \\
\text { cN+ } \\
\text { Initial Stage } \\
\text { I } \\
\text { II } \\
\text { III } \\
\text { IV } \\
\text { Initial Treatment } \\
\text { RT } \\
\text { CRT } \\
\text { Initial Treatment Site } \\
\text { OSH } \\
\text { UM }\end{array}$ & $\begin{array}{l}59.2(10.1) \\
143(58.6) \\
98(40.1) \\
0(0) \\
63(25.8) \\
71(29.0) \\
65(26.6) \\
28(11.4) \\
188(77.0) \\
40(16.3) \\
63(25.8) \\
62(25.4) \\
63(25.8) \\
39(15.9) \\
139(56.9) \\
105(43.0) \\
192(78.6) \\
52(21.3)\end{array}$ & $\begin{array}{l}16(6.5) \\
17(6.9)\end{array}$ \\
\hline $\begin{array}{l}\text { Recurrent Cancer } \\
\text { Age at Recurrence (yrs) } \\
\text { Time to Recurrence (mo) } \\
\text { Time to Recurrence < 2yr } \\
\text { Yes } \\
\text { No } \\
\text { Recurrent Site }\end{array}$ & $\begin{array}{l}61.2(10.0) \\
21.4(30.2) \\
126(51.6) \\
118(48.4)\end{array}$ & $\begin{array}{l}0(0) \\
0(0) \\
0(0)\end{array}$ \\
\hline
\end{tabular}




\begin{tabular}{|l|l|l|}
\hline Glottis & $136(55.7)$ & $0(0)$ \\
Supraglottis & $108(44.2)$ & \\
Recurrent cT Classification & $14(5.7)$ & $0(0)$ \\
CT1 & $97(39.7)$ & \\
cT2 & $65(26.6)$ & \\
CT3 & $68(27.8)$ & \\
cT4 & & $0(0)$ \\
Recurrent cN Classification & $215(88.1)$ & \\
CN0 & $29(11.8)$ & $0(0)$ \\
CN+ & $13(5.3)$ & \\
Recurrent cStage & $93(38.1)$ & \\
I & $62(25.4)$ & \\
II & $76(31.1)$ & \\
III & IV & \\
\hline
\end{tabular}

*Percentages calculated including the missing values where applicable. Smoking status is defined as current (smoking within one year prior to surgery), or former (quit greater than one year prior to surgery).

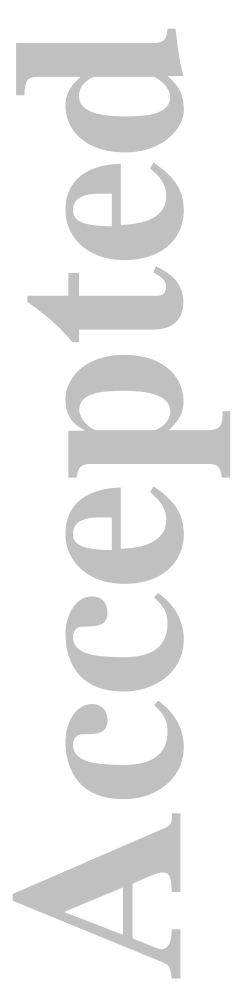

John Wiley \& Sons, Inc.

This article is protected by copyright. All rights reserved. 
Table 2. Perioperative Complications in Patients Undergoing Surgery for Recurrent/Persistent LSCC.

\begin{tabular}{|l|l|l|l|}
\hline Comorbidity & Fistula & $\begin{array}{l}\text { OR } \\
\text { takeback }\end{array}$ & $\begin{array}{l}\text { 30-day } \\
\text { mortality }\end{array}$ \\
\hline None & $39 \%$ & $12 \%$ & $0 \%$ \\
\hline Mild/Moderate & $33 \%$ & $12 \%$ & $4 \%$ \\
\hline Severe & $40 \%$ & $20 \%$ & $7 \% *$ \\
\hline
\end{tabular}

${ }^{*} p=0.001$ comparing severe versus none comorbidity status.

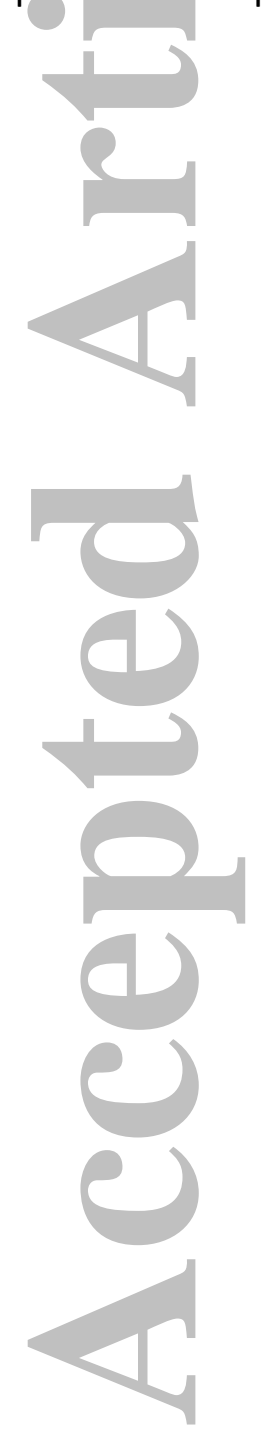

John Wiley \& Sons, Inc.

This article is protected by copyright. All rights reserved. 
Table 3. Multivariable Preoperative Predictors of Survival for Recurrent/Persistent LSCC.

\begin{tabular}{|c|c|c|c|}
\hline Characteristic & $\begin{array}{l}\text { OS } \\
\text { HR }(95 \% \mathrm{Cl})\end{array}$ & $\begin{array}{l}\text { DSS } \\
\text { HR (95\% Cl) }\end{array}$ & $\begin{array}{l}\text { DFS } \\
\text { HR }(95 \% \mathrm{Cl})\end{array}$ \\
\hline $\begin{array}{l}\text { Recurrent cN+ } \\
\text { No (ref) } \\
\text { Yes } \\
\end{array}$ & $2.91(1.74-4.88)$ & $\begin{array}{l}-- \\
3.95(2.16-7.22)\end{array}$ & $3.26(1.83-5.78)$ \\
\hline $\begin{array}{l}\text { Initial Stage } \\
\text { I (ref) } \\
\text { II } \\
\text { III } \\
\text { IV }\end{array}$ & $\begin{array}{l}- \\
2.96(1.59-5.51) \\
3.40(1.82-6.34) \\
1.73(0.81-3.66)\end{array}$ & $\begin{array}{l}-- \\
2.62(1.15-5.98) \\
2.13(0.82-5.53) \\
0.99(0.31-3.15)\end{array}$ & $\begin{array}{l}-\overline{2.36}(1.12-4.97) \\
3.14(1.54-6.39) \\
1.71(0.73-4.00)\end{array}$ \\
\hline $\begin{array}{l}\text { Comorbidities } \\
\text { None (ref) } \\
\text { Mild/Moderate } \\
\text { Severe }\end{array}$ & $\begin{array}{l}-\overline{1.43}(0.86-2.38) \\
\mathbf{3 . 7 6}(\mathbf{1 . 5 6}-\mathbf{9 . 0 6})\end{array}$ & $\begin{array}{l}-- \\
-- \\
--\end{array}$ & $\begin{array}{l}-- \\
-- \\
--\end{array}$ \\
\hline
\end{tabular}
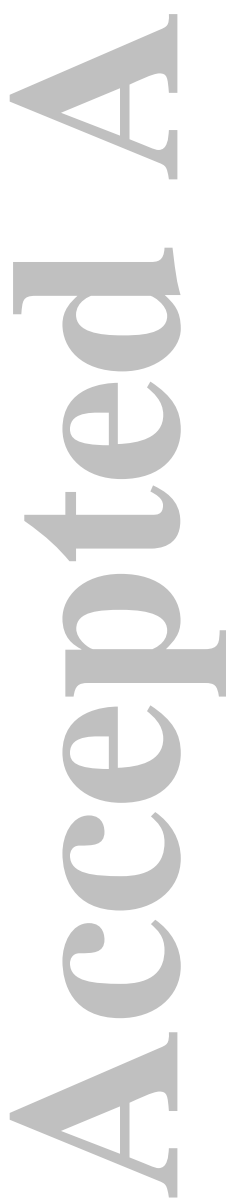

John Wiley \& Sons, Inc.

This article is protected by copyright. All rights reserved. 